PROCEEDINGS OF THE

AMERICAN MATHEMATICAL SOCIETY

Volume 130, Number 11, Pages 3369-3377

S 0002-9939(02)06473-0

Article electronically published on April 17, 2002

\title{
TOPOLOGICALLY TRANSVERSAL REVERSIBLE HOMOCLINIC SETS
}

\author{
MICHAL FEČKAN
}

(Communicated by Carmen Chicone)

\begin{abstract}
An $R$-reversible diffeomorphism on $\mathbb{R}^{2 N}$ is studied possessing a hyperbolic fixed point. If the stable manifold of the hyperbolic fixed point and the fixed point set Fix $R$ of $R$ have a nontrivial local topological crossing, then an infinite number of $R$-symmetric periodic orbits of the diffeomorphism is shown. A perturbed problem is also studied by showing the relationship between a corresponding Melnikov function and the nontriviality of a local topological crossing of the set Fix $R$ and the stable manifold for the perturbed diffeomorphism.
\end{abstract}

\section{INTRODUCTION}

Let $R: \mathbb{R}^{2 N} \rightarrow \mathbb{R}^{2 N}$ be a linear involution, i.e. $R^{2}=I$, such that $\operatorname{dim}$ Fix $R=N$, where Fix $R=\left\{x \in \mathbb{R}^{2 N} \mid R x=x\right\}$. Consider a $C^{1}$-smooth diffeomorphism $f: \mathbb{R}^{2 N} \rightarrow \mathbb{R}^{2 N}$ which is $R$-reversible, i.e. $R f(x)=f^{-1}(R x), \forall x \in \mathbb{R}^{2 N}$, and possessing an $R$-symmetric hyperbolic fixed point $p \in$ Fix $R$. Any subset of $\mathbb{R}^{2 N}$ invariant under the action of $R$ is called $R$-symmetric. Reversible diffeomorphisms naturally come from mechanics [5] as the time flow mappings of second order gradient differential equations. Let $W_{p}^{s}, W_{p}^{u}$ be the global stable and unstable manifolds of $p$, respectively. Let $\widetilde{W}_{p}^{s}$ be an open subset of $W_{p}^{s}$ which is a submanifold of $\mathbb{R}^{2 N}$, i.e. the immersed and induced topologies on $\widetilde{W}_{p}^{s}$ coincide, and such that $\widetilde{W}_{p}^{s} \backslash\{p\} \cap$ Fix $R \neq \emptyset$, i.e. there is an $R$-symmetric point $q$ homoclinic to $p$ 10]. Since $R W_{p}^{s}=W_{p}^{u}$, we put $\widetilde{W}_{p}^{u}=R \widetilde{W}_{p}^{s}$. We also suppose the existence of a compact component $K \ni q$ of the set $\widetilde{W}_{p}^{s} \cap$ Fix $R$, that is a compact subset $K \subset \widetilde{W}_{p}^{s} \backslash\{p\} \cap$ Fix $R$ such that $q \in K$ and there exists an open bounded subset $U \subset \bar{U} \subset \mathbb{R}^{2 N} \backslash\{p\}$ satisfying $U \cap \widetilde{W}_{p}^{s} \cap$ Fix $R=K$. By shrinking $U$, we can assume that $\overline{W_{p}^{s} \cap U}=\widetilde{W}_{p}^{s} \cap \bar{U}$. We note that $\widetilde{W}_{p}^{s} \cap U$ is an orientable submanifold of $\mathbb{R}^{2 N}$. Then we can define the local intersection number $\#\left(\widetilde{W}_{p}^{s} \cap U\right.$, Fix $\left.R \cap U\right)$ of the stable manifold $W_{p}^{s}$ and the plain Fix $R$ in $U \subset \mathbb{R}^{2 N}$ [7. The main purpose of this note is to prove the following result.

Theorem 1.1. If \# $\left(\widetilde{W}_{p}^{s} \cap U\right.$, Fix $\left.R \cap U\right) \neq 0$, then there is an $\omega_{0} \in \mathbb{N}$ such that for any $\mathbb{N} \ni \omega \geq \omega_{0}$ the diffeomorphism $f$ possesses a $2 \omega$-periodic orbit $\left\{x_{n}^{\omega}\right\}_{n \in \mathbb{Z}}$ such

Received by the editors April 25, 2001 and, in revised form, June 29, 2001.

2000 Mathematics Subject Classification. Primary 37C25, 37C29, 57R50.

The author was partially supported by Grant GA-MS 1/6179/00. 
that $R x_{n}^{\omega}=x_{-n}^{\omega}, n \in \mathbb{Z}$. Moreover, $x_{0}^{\omega} \in$ Fix $R$ is near the set $K$, while $x_{\omega}^{\omega} \in$ Fix $R$ is near the point $p$.

When $q$ is a transversal intersection of $W_{p}^{s}$ and Fix $R$, then Theorem 1.1 is proved in 3], 4], [5], 9] and [10]. Then clearly $\#\left(\widetilde{W}_{p}^{s} \cap U\right.$, Fix $\left.R \cap U\right) \neq 0$ for a small open neighbourhood $U$ of $q$. Furthermore, we study the case where $W_{p}^{s}$ and Fix $R$ intersect on a compact manifold. Then we consider a $C^{2}$-smooth $R$-reversible perturbation of $f$. Associated to such a perturbation there is a Melnikov function. We show that if the Brouwer degree [6] of this Melnikov function is not zero, and the perturbation is small, then the perturbed stable manifold $W_{p, p e r}^{s}$ and the plain Fix $R$ satisfy \# $\left(\widetilde{W}_{p, p e r}^{s} \cap U\right.$, Fix $\left.R \cap U\right) \neq 0$ with the corresponding infinitely many $R$-symmetric periodic orbits of the perturbed diffeomorphism. Finally, we note that any accumulation point of the set $\left\{x_{0}^{\omega}\right\}_{\omega \geq \omega_{0}} \subset$ Fix $R$ from Theorem 1.1 is a starting point of an $R$-symmetric homoclinic orbit of $f$ to $p$.

If $p$ is a hyperbolic fixed point of $f$ but not $R$-symmetric, then $R p$ is also a hyperbolic fixed point of $f$. If $q \in W_{p}^{s} \cap$ Fix $R$, then $q \in W_{p}^{s} \cap W_{R p}^{u}$ [5], hence $q$ lies on an $R$-symmetric heteroclinic orbit connecting $p$ and $R p$. Consequently, as for Theorem 1.1, we can prove the following result.

Theorem 1.2. Suppose $f$ has a non-R-symmetric hyperbolic fixed point $p$. If $W_{p}^{s}$ and $W_{p}^{u}$ meet Fix $R$ locally topologically transversally, then $f$ has an infinite number of $R$-symmetric periodic orbits with periods tending to infinity.

We note that for the case $N=1$ it is elementary to show that if $p$ is a hyperbolic fixed point of $f$ and $W_{p}^{s}$ (or $W_{p}^{u}$ ) meets Fix $R$, then a local intersection number of $W_{p}^{s}$ (or $W_{p}^{u}$ ) with Fix $R$ is nonzero. Then Theorems 1.1 and 1.2 can be applied. Indeed, let $q \in W_{p}^{s} \cap$ Fix $R$ be the first intersection starting on $W_{p}^{s}$ from $p$. Then the points $f^{-1}(q), f(q) \in W_{p}^{s}$ lie on the opposite half-plains separated by Fix $R$. Hence an open bounded connected part $\widetilde{W}_{p}^{s}$ of $W_{p}^{s}$ such that $f^{-1}(q), f(q) \in \widetilde{W}_{p}^{s}$ topologically nontrivially crosses Fix $R$. Similarly for $W_{p}^{u}$.

The paper is finished with an example of a perturbed second order differential equation in $\mathbb{R}^{2}$ with a topologically transversal, but non- $C^{1}$-transversal, intersection of the stable manifold and Fix $R$.

\section{Preliminary Results}

Let $(\cdot, \cdot)$ be an inner product on $\mathbb{R}^{2 N}$. By following [10], we set

$$
\langle x, y\rangle=\frac{1}{2}((x, y)+(R x, R y)) .
$$

Then $\langle R x, R y\rangle=\langle x, y\rangle, x, y \in \mathbb{R}^{2 N}$, and so $\|R\|=\left\|R^{-1}\right\|=1$. Since $R K=K$, we can assume that $R U=U$.

For any $\xi \in \widetilde{W}_{p}^{s} \cap \bar{U}$ we set $\xi_{n}=f^{n}(\xi), n \in \mathbb{Z}_{+}, \eta_{n}=f^{n}(\eta), n \in \mathbb{Z}_{-}, \eta=R \xi$, where $\mathbb{Z}_{+}=\{0,1,2, \cdots\}$ and $\mathbb{Z}_{-}=\{\cdots,-2,-1,0\}$. We note that $\eta_{-n}=R \xi_{n}$, $n \in \mathbb{Z}_{+}$. Then the linear system

$$
v_{n+1}=\operatorname{Df}\left(\xi_{n}\right) v_{n}, \quad n \in \mathbb{Z}_{+},
$$

has an exponential dichotomy on $\mathbb{Z}_{+}$[8], i.e. there are positive constants $L, \delta \in$ $(0,1)$ and the orthogonal projection $P_{\xi}: \mathbb{R}^{2 N} \rightarrow T_{\xi} \widetilde{W}_{p}^{s}$ such that the fundamental 
solution $V_{\xi}(n)$ of (1) satisfies the following:

$$
\begin{aligned}
& \left\|V_{\xi}(n) P_{\xi} V_{\xi}(m)^{-1}\right\| \leq L \delta^{n-m}, \quad m \leq n, \quad m, n \in \mathbb{Z}_{+}, \\
& \left\|V_{\xi}(n)\left(I-P_{\xi}\right) V_{\xi}(m)^{-1}\right\| \leq L \delta^{m-n}, \quad n \leq m, \quad m, n \in \mathbb{Z}_{+} .
\end{aligned}
$$

We note that $L$ and $\delta$ can be chosen to be independent of $\xi \in \widetilde{W}_{p}^{s} \cap \bar{U}$ [1].

By defining $R v_{n}=w_{-n}$ in (1), the reversibility of $f$ implies

$$
w_{n+1}=D f\left(\eta_{n}\right) w_{n}, \quad n \in \mathbb{Z}_{-}, \quad n \neq 0 .
$$

Hence, the fundamental solution $W_{\xi}(n)$ of (2) is given by $W_{\xi}(n)=R V_{\xi}(-n) R^{-1}$, $n \in \mathbb{Z}_{-}$, and since $\|R\|=\left\|R^{-1}\right\|=1$, then (2) has an exponential dichotomy on $\mathbb{Z}_{-}$ with the constants $L, \delta$ and the orthogonal projection $I-Q_{\eta}$, where $Q_{\eta}=R P_{\xi} R^{-1}$. We note $\eta=R \xi$.

Now we fix $\omega \in \mathbb{N}$ large and put

$$
\begin{aligned}
& J_{\omega}=\{-\omega,-\omega+1, \cdots, \omega-1, \omega\}, \\
& J_{\omega}^{-}=\{-\omega,-\omega+1, \cdots,-1,0\}, \quad I_{\omega}^{-}=\{-\omega,-\omega+1, \cdots,-1,\}, \\
& J_{\omega}^{+}=\{0,1, \cdots, \omega-1, \omega\}, \quad I_{\omega}^{+}=\{0,1, \cdots, \omega-2, \omega-1\} .
\end{aligned}
$$

We note that the family $\left\{P_{\xi} \mid \xi \in \widetilde{W}_{p}^{s} \cap U\right\}$ is continuous on $\widetilde{W}_{p}^{s} \cap U$. In this paper, $\mathcal{R} L$ and $\mathcal{N} L$ denote, respectively, the range and the kernel of a linear operator $L$.

Theorem 2.1 ([1]). There exist $\omega_{0} \in \mathbb{N}$ and a constant $c>0$ such that, for any $\omega \in \mathbb{N}, \omega \geq \omega_{0}$, and $\xi \in \widetilde{W}_{p}^{s} \cap U$, there exist unique $\left\{x_{n}^{+}(\omega, \xi)\right\}_{n \in J_{\omega}^{+}}$and $\left\{x_{n}^{-}(\omega, \xi)\right\}_{n \in J_{\omega}^{-}}$which satisfy $x_{n+1}=f\left(x_{n}\right)$ separately on $I_{\omega}^{+}$and $I_{\omega}^{-}$such that

$$
\begin{aligned}
& P_{\xi} x_{0}^{+}(\omega, \xi)=P_{\xi} \xi, \quad Q_{R \xi} x_{0}^{-}(\omega, \xi)=Q_{R \xi} R \xi, \\
& x_{\omega}^{+}(\omega, \xi)=x_{-\omega}^{-}(\omega, \xi)
\end{aligned}
$$

together with

$$
\begin{aligned}
& \max _{n \in J_{\omega}^{+}}\left|x_{n}^{+}(\omega, \xi)-\xi_{n}\right| \leq c \delta^{\omega} \\
& \max _{n \in J_{\omega}^{-}}\left|x_{n}^{-}(\omega, \xi)-\eta_{n}\right| \leq c \delta^{\omega}
\end{aligned}
$$

Moreover, $x_{n}^{ \pm}(\omega, \xi)$ are continuous with respect to $\xi$.

Proof. We study the nonlinear system

$$
x_{n+1}=f\left(x_{n}\right)
$$

near $\left\{\xi_{n}\right\}_{n \in J_{\omega}^{+}}$and $\left\{\eta_{n}\right\}_{n \in J_{\omega}^{-}}$. By putting $x_{n}^{+}=\xi_{n}+v_{n}, n \in J_{\omega}^{+}$and $x_{n}^{-}=\eta_{n}+w_{n}$, $n \in J_{\omega}^{-}$, we get the systems

$$
v_{n+1}=D f\left(\xi_{n}\right) v_{n}+f\left(\xi_{n}+v_{n}\right)-f\left(\xi_{n}\right)-D f\left(\xi_{n}\right) v_{n}
$$

$$
=D f\left(\xi_{n}\right) v_{n}+o\left(\left|v_{n}\right|\right), \quad n \in I_{\omega}^{+}
$$

and

$$
w_{n+1}=D f\left(\eta_{n}\right) w_{n}+f\left(\eta_{n}+w_{n}\right)-f\left(\eta_{n}\right)-D f\left(\eta_{n}\right) w_{n}
$$

$$
=D f\left(\eta_{n}\right) w_{n}+o\left(\left|w_{n}\right|\right), \quad n \in I_{\omega}^{-}
$$


Since we are looking for solutions of equation (3) such that $x_{\omega}^{+}=x_{-\omega}^{-}$, we add the boundary value conditions

$$
v_{\omega}-w_{-\omega}=\eta_{-\omega}-\xi_{\omega}=O\left(\delta^{\omega}\right), \quad P_{\xi} v_{0}=0, \quad Q_{R \xi} w_{0}=0 .
$$

Let $v=\left(v_{0}, \ldots, v_{\omega}\right) \in \mathbb{R}^{N(\omega+1)}, w=\left(w_{-\omega}, \ldots, w_{0}\right) \in \mathbb{R}^{N(\omega+1)}$. To solve equations (4)-(6), we take the mapping $\Gamma_{\omega}: \widetilde{W}_{p}^{s} \cap \bar{U} \times \mathbb{R}^{2 N(\omega+1)} \rightarrow \mathbb{R}^{2 N(\omega+1)}$ defined by

$$
\Gamma_{\omega}(\xi, v, w)=\left(\begin{array}{c}
\left(v_{n+1}-f\left(\xi_{n}+v_{n}\right)+f\left(\xi_{n}\right)\right)_{n \in I_{\omega}^{+}} \\
\left(w_{n+1}-f\left(\eta_{n}+w_{n}\right)+f\left(\eta_{n}\right)\right)_{n \in I_{\omega}^{-}} \\
v_{\omega}-w_{-\omega}-\left(\eta_{-\omega}-\xi_{\omega}\right) \\
P_{\xi} v_{0} \\
Q_{R \xi} w_{0}
\end{array}\right) .
$$

where $\left(\begin{array}{c}P_{\xi} v_{0} \\ Q_{R \xi} w_{0}\end{array}\right)$ has to be meant as a vector in $\mathbb{R}^{N}=\mathcal{R} P_{\xi} \times \mathcal{R} Q_{R \xi}$. We already know that $P_{\xi}$ and $Q_{R \xi}$ are continuous. Thus, for any fixed $\omega \geq \omega_{0}, \Gamma_{\omega}$ is continuous in $(\xi, u, v)$ as well as its derivatives with respect to $(v, w)$ when we take on $\mathbb{R}^{2 N(\omega+1)}$ the maximum norm $\max _{i}\left\{\left|v_{i}\right|,\left|w_{i}\right|\right\}$. We have $\Gamma_{\omega}(\xi, 0,0)=O\left(\delta^{\omega}\right)$ uniformly with respect to $\xi$ and the linearized map $D_{(v, w)} \Gamma_{\omega}(\xi, 0,0)$ has the form

$$
D_{(v, w)} \Gamma_{\omega}(\xi, 0,0)\left(\begin{array}{c}
v \\
w
\end{array}\right)=\left(\begin{array}{c}
\left(v_{n+1}-D f\left(\xi_{n}\right) v_{n}\right)_{n \in I_{\omega}^{+}} \\
\left(w_{n+1}-D f\left(\eta_{n}\right) w_{n}\right)_{n \in I_{\omega}^{-}} \\
v_{\omega}-w_{-\omega} \\
P_{\xi} v_{0} \\
Q_{R \xi} w_{0} .
\end{array}\right) .
$$

Arguing as in Lemma 2.1 of [1] or Lemma 2 of [2], the map $D_{(v, w)} \Gamma_{\omega}(\xi, 0,0)$ is invertible and that its inverse is bounded uniformly with respect to $\xi$. Hence from the implicit function theorem we get that $c>0$ and $\omega_{0} \gg 1$ exist such that for $\omega \geq \omega_{0}$, the equation $\Gamma_{\omega}(\xi, v, w)=0$ can be solved uniquely for $(v, w)$ in a neighborhood of $(0,0)$ in terms of $(\xi, \omega)$. Moreover $\max _{i}\left\{\left|v_{i}\right|,\left|w_{i}\right|\right\}<c \delta^{\omega}$, and the solution is continuous in $\xi$, for any fixed $\omega \geq \omega_{0}$. The proof is finished.

Since $Q_{R \xi}=R P_{\xi} R^{-1}, \eta_{-n}=R \xi_{n}, n \in \mathbb{Z}_{+}$, we see that the sequences given by $y_{n}^{-}(\omega, \xi)=R x_{-n}^{+}(\omega, \xi), n \in J_{\omega}^{-}, y_{n}^{+}(\omega, \xi)=R x_{-n}^{-}(\omega, \xi), n \in J_{\omega}^{+}$also satisfy the statement of Theorem 2.1. The uniqueness of such orbits implies that

$$
\begin{array}{ll}
x_{n}^{+}(\omega, \xi)=y_{n}^{+}(\omega, \xi)=R x_{-n}^{-}(\omega, \xi), & n \in J_{\omega}^{+}, \\
x_{n}^{-}(\omega, \xi)=y_{n}^{-}(\omega, \xi)=R x_{-n}^{+}(\omega, \xi), & n \in J_{\omega}^{-} .
\end{array}
$$

Hence $R x_{n}^{ \pm}(\omega, \xi)=x_{-n}^{\mp}(\omega, \xi), n \in J_{\omega}^{ \pm}$. So the orbit of $f$ in Theorem 2.1 is $R$ symmetric.

\section{3. $R$-SYMMETRIC PERIODIC ORBITS}

In this section we prove Theorem 1.1. If $x_{0}^{+}(\omega, \xi) \in$ Fix $R$, then

$$
x_{0}^{+}(\omega, \xi)=R x_{0}^{+}(\omega, \xi)=x_{0}^{-}(\omega, \xi) .
$$

Consequently, the orbit of Theorem 2.1 becomes an $R$-symmetric periodic orbit of $f$. Hence we have to solve the equation

$$
(I-R) x_{0}^{+}(\omega, \xi)=0, \quad \xi \in \widetilde{W}_{p}^{s} \cap U .
$$


Let $V$ be an open subset such that $K \subset V \subset \bar{V} \subset U$ and let $\omega_{0}$ be as in Theorem 2.1. Note that the solution $x_{0}^{+}(\omega, \xi)$ is defined for $\xi \in \widetilde{W}_{p}^{s} \cap \bar{V}$ and

$$
\#\left(\widetilde{W}_{p}^{s} \cap V, \text { Fix } R \cap V\right)=\#\left(\widetilde{W}_{p}^{s} \cap U, \operatorname{Fix} R \cap U\right) \neq 0 .
$$

To solve (7), we put $F_{\omega}(\xi)=(I-R) x_{0}^{+}(\omega, \xi), F_{\omega}: \widetilde{W}_{p}^{s} \cap \bar{V} \rightarrow R_{-}=\mathcal{R}(I-R)$. We note that $\operatorname{dim} R_{-}=\operatorname{dim} \widetilde{W}_{p}^{s}$.

Now we put $F_{\omega}$ into the homotopy

$$
\begin{aligned}
& H_{\omega}: \widetilde{W}_{p}^{s} \cap \bar{V} \times[0,1] \rightarrow R_{-}, \\
& H_{\omega}(\xi, \lambda)=\lambda F_{\omega}(\xi)+(1-\lambda)(I-R) \xi
\end{aligned}
$$

Theorem 2.1 gives

$$
\left|H_{\omega}(\xi, \lambda)-(I-R) \xi\right|=\left|\lambda\left(F_{\omega}(\xi)-(I-R) \xi\right)\right| \leq c \delta^{\omega} .
$$

Consequently, $H_{\omega}(\cdot, \lambda) \neq 0$ on the boundary $\partial\left(\widetilde{W}_{p}^{s} \cap V\right)$ for any $0 \leq \lambda \leq 1$. This gives for the Brouwer degree [6],

$$
\operatorname{deg}\left(F_{\omega}, \widetilde{W}_{p}^{s} \cap V, 0\right)= \pm \#\left(\widetilde{W}_{p}^{s} \cap V, \text { Fix } R \cap V\right) \neq 0 .
$$

Summarizing, we see that $F_{\omega}(\xi)=0$ has a solution $\xi \in \widetilde{W}_{p}^{s} \cap V$ for any $\omega \geq \omega_{0}$, where $\omega_{0}$ is sufficiently large. This proves Theorem 1.1.

\section{Perturbation theory}

In this section, we consider a $C^{2}$-smooth perturbation $f(x, \varepsilon)$ of $f$, i.e. we suppose that $f(x, 0)=f(x)$ and $R f(x, \varepsilon)=f^{-1}(R x, \varepsilon), \forall x \in \mathbb{R}^{2 N}, \varepsilon$ small. Then Theorem 2.1 gives a $C^{1}$-mapping $x_{0}^{+}(\omega, \xi, \varepsilon)$ and we are led to the equation

$$
(I-R) x_{0}^{+}(\omega, \xi, \varepsilon)=0, \quad \xi \in \widetilde{W}_{p}^{s} \cap U .
$$

By taking $\omega \rightarrow \infty$ in the above equation, we get $F(\xi, \varepsilon)=(I-R) x_{0}^{+}(\infty, \xi, \varepsilon)=0$ which is precisely the equation of $R$-symmetric homoclinic solutions to the hyperbolic symmetric fixed point $p_{\varepsilon}$ of $f(x, \varepsilon)$ near $p$ [10]. We assume that this equation has a compact nondegenerate solution manifold, i.e.

(H1) There is an embedded compact $C^{2}$-smooth submanifold $\mathcal{M} \subset \widetilde{W}_{p}^{s} \backslash\{p\} \cap$ Fix $R$ for an open subset $\widetilde{W}_{p}^{s}$ of $W_{p}^{s}$ which is a submanifold of $\mathbb{R}^{2 N}$ and such that $\operatorname{dim} \mathcal{N}(I-R) D_{\xi} x_{0}^{+}(\infty, \xi, 0)=\operatorname{dim} \mathcal{M}$ for any $\xi \in \mathcal{M}$. Furthermore, let $\mathcal{O} \subset \widetilde{W}_{p}^{s} \backslash\{p\}$ be an open bounded subset such that $\mathcal{M} \subset \mathcal{O}$. Then $\mathcal{O}$ can be oriented. We suppose that $\mathcal{M}$ is orientable embedded into $\mathcal{O}$.

We note that always $T_{\xi} \mathcal{M} \subset \mathcal{N}(I-R) D_{\xi} x_{0}^{+}(\infty, \xi, 0), \forall \xi \in \mathcal{M}$ and $\operatorname{dim} \mathcal{M}=$ $\operatorname{dim} T_{\xi} \mathcal{M}$, hence (H1) implies $T_{\xi} \mathcal{M}=\mathcal{N}(I-R) D_{\xi} x_{0}^{+}(\infty, \xi, 0), \forall \xi \in \mathcal{M}$. Since $x_{0}^{+}(\infty, \xi, 0)=\xi$, we have $D_{\xi} x_{0}^{+}(\infty, \xi, 0) v=v, v \in T_{\xi} \widetilde{W}_{p}^{s}$. Hence

$$
\mathcal{N}(I-R) D_{\xi} x_{0}^{+}(\infty, \xi, 0)=\text { Fix } R \cap T_{\xi} \widetilde{W}_{p}^{s} .
$$

Now we take a tubular neighbourhood $\mathcal{V}$ of $\mathcal{M}$ in $\widetilde{W}_{p}^{s}$, i.e. any $\xi \in \mathcal{V}$ can be uniquely expressed as a pair $(\tau, v)$, where $\tau \in \mathcal{M}$ and $v \in T_{\tau} \widetilde{W}_{p}^{s} / T_{\tau} \mathcal{M}=$ $T_{\tau} \widetilde{W}_{p}^{s} /\left(\right.$ Fix $\left.R \cap T_{\tau} \widetilde{W}_{p}^{s}\right)=N_{\tau}$-the fiber of the normal vector bundle of $\mathcal{M}$ in $\widetilde{W}_{p}^{s}$, and $|v|<\Delta$ for some $\Delta>0$. Hence we identify $\mathcal{V}$ with an open neighbourhood of the zero section of the normal vector bundle of $\mathcal{M}$ in $\widetilde{W}_{p}^{s}$. Let $S_{\tau}: \operatorname{Fix}(-R) \rightarrow$ $\mathcal{R} D_{v} F(\tau, 0,0)$ be the orthogonal projection. We note that the assumption (H1) 
implies the invertibility of the linear mapping $D_{v} F(\tau, 0,0): N_{\tau} \rightarrow \mathcal{R} D_{v} F(\tau, 0,0)$, since $D_{v} F(\tau, 0,0) w=(I-R) w, w \in N_{\tau}$.

From $F(\tau, 0,0)=0$, we get $F(\tau, v, \varepsilon)=D_{v} F(\tau, 0,0) v+\varepsilon D_{\varepsilon} F(\tau, 0,0)+o(|v|)+$ $o(\varepsilon)$. We consider the homotopy

$$
\begin{aligned}
H(\tau, v, \varepsilon, \lambda)= & S_{\tau}\left(\lambda F(\tau, v, \varepsilon)+(1-\lambda) D_{v} F(\tau, 0,0) v\right) \\
& +\left(I-S_{\tau}\right)\left(\lambda F(\tau, v, \varepsilon)+(1-\lambda) \varepsilon D_{\varepsilon} F(\tau, 0,0)\right) .
\end{aligned}
$$

Then $H(\tau, v, \varepsilon, 0)=S_{\tau} D_{v} F(\tau, 0,0) v+\varepsilon\left(I-S_{\tau}\right) D_{\varepsilon} F(\tau, 0,0)$ and $H(\tau, v, \varepsilon, 1)=$ $F(\tau, v, \varepsilon)$.

Now we suppose

(H2) There is an open connected subset $\Omega \subset \mathcal{M}$ such that $B(\tau) \neq 0, \forall \tau \in \partial \Omega$, where $B(\tau)=\left(I-S_{\tau}\right) D_{\varepsilon} F(\tau, 0,0)$.

Since $\mathcal{M}$ is orientable embedded into $\mathcal{O}$ and $\mathcal{O}$ is orientable, the tangent vector bundle $T \mathcal{M}$ and the normal vector bundle $\bigcup_{\tau \in \mathcal{M}} N_{\tau}$ are both orientable. Hence the vector bundle $\bigcup_{\tau \in \mathcal{M}}(I-R) N_{\tau}=\bigcup_{\tau \in \mathcal{M}} \mathcal{R} D_{v} F(\tau, 0,0)$ is also orientable, because $D_{v} F(\tau, 0,0): N_{\tau} \rightarrow \mathcal{R} D_{v} F(\tau, 0,0)$ is invertible. Since $S_{\tau}$ : $\operatorname{Fix}(-R) \rightarrow \mathcal{R} D_{v} F(\tau, 0,0)$ is the orthogonal projection and the vector bundle $\bigcup_{\tau \in \mathcal{M}} \operatorname{Fix}(-R)=\mathcal{M} \times \operatorname{Fix}(-R)$ is orientable, we get the orientability of the vector bundle $\bigcup_{\tau \in \mathcal{M}} \mathcal{R}\left(I-S_{\tau}\right) \operatorname{Fix}(-R)$. Since $B(\tau)$ is a section of this vector bundle, the following assumption makes sense [7].

(H3) $\operatorname{deg}(B(\tau), \Omega, 0) \neq 0$.

According to (H2), there is an open connected bounded neighbourhood $U_{1} \subset \mathcal{M}$ of $\bar{\Omega}$ such that $B(\lambda) \neq 0, \forall \tau \in U_{1} \backslash \Omega$. Now we take an open subset $V_{\varepsilon}=\{(\tau, v) \in$ $V \mid \tau \in U_{1}$ and $\left.|v|<|\varepsilon| r_{1}\right\}$ for a positive constant $r_{1}$ and $0<|\varepsilon|<\triangle / r_{1}$. Since

$$
S_{\tau}\left(\lambda F(\tau, v, \varepsilon)+(1-\lambda) D_{v} F(\tau, 0,0) v\right)=S_{\tau} D_{v} F(\tau, 0,0) v+o(|v|)+O(\varepsilon)
$$

and $S_{\tau} D_{v} F(\tau, 0,0): N_{\tau} \rightarrow \mathcal{R} D_{v} F(\tau, 0,0)$ is invertible, we get that

$$
S_{\tau}\left(\lambda F(\tau, v, \varepsilon)+(1-\lambda) D_{v} F(\tau, 0,0) v\right) \neq 0,
$$

$\forall(\tau, v) \in V_{\varepsilon},|v|=r_{1}|\varepsilon|$ for a $r_{1}$ sufficiently large and fixed. Furthermore, we have $\left(I-S_{\tau}\right)\left(\lambda F(\tau, v, \varepsilon)+(1-\lambda) \varepsilon D_{\varepsilon} F(\tau, 0,0)\right)=\varepsilon\left(I-S_{\tau}\right) D_{\varepsilon} F(\tau, 0,0)+o(|v|)+o(\varepsilon)$

since $\left(I-S_{\tau}\right) D_{v} F(\tau, 0,0)=0$. Hence $\left(I-S_{\tau}\right)\left(\lambda F(\tau, v, \varepsilon)+(1-\lambda) \varepsilon D_{\varepsilon} F(\tau, 0,0)\right) \neq 0$ for $(\tau, v) \in V_{\varepsilon},|v| \leq r_{1}|\varepsilon|$ and $\tau \in U_{1} \backslash \Omega$.

Summarizing, we see that $H(\tau, v, \varepsilon, \lambda) \neq 0$ for any $(\tau, v) \in \partial V_{\varepsilon}, \lambda \in[0,1]$ and $\varepsilon \neq 0$ sufficiently small. Consequently [] ,

$$
\operatorname{deg}\left(F, V_{\varepsilon}, 0\right)=\operatorname{deg}\left(H(\cdot, \varepsilon, 0), V_{\varepsilon}, 0\right),
$$

where $H(\tau, v, \varepsilon, 0)=S_{\tau} D_{v} F(\tau, v(\tau, \varepsilon), \varepsilon) v+\varepsilon\left(I-S_{\tau}\right) D_{\varepsilon} F(\tau, 0,0)$. Since the linear map $S_{\tau} D_{v} F(\tau, v(\tau, \varepsilon), \varepsilon): N_{\tau} \rightarrow \mathcal{R} D_{v} F(\tau, 0,0)$ is invertible and $U_{1}$ is connected, we get

$$
\operatorname{deg}\left(H(\cdot, \varepsilon, 0), V_{\varepsilon}, 0\right)= \pm \operatorname{deg}(B(\tau), \Omega, 0) \neq 0 .
$$

This implies \#( $\widetilde{W}_{p_{\varepsilon}}^{s} \cap V_{\varepsilon}$, Fix $\left.R \cap V_{\varepsilon}\right) \neq 0$. Summarizing we get the following result.

Theorem 4.1. Assume (H1), (H2) and (H3). Then there exists $\varepsilon_{0}>0$ such that for $0<|\varepsilon| \leq \varepsilon_{0}$, it is nonzero the local intersection number of the plain Fix $R$ and the stable manifold of the hyperbolic fixed point of the map $x_{n+1}=f\left(x_{n}, \varepsilon\right)$ which is located near the fixed point $p$ of the map $x_{n+1}=f\left(x_{n}\right)$. 
Hence Theorem 1.1 and the assumptions of Theorem 4.1 imply an infinite number of $R$-symmetric periodic orbits of $f(x, \varepsilon)$ accumulating on $R$-symmetric homoclinic orbits of $f(x, \varepsilon)$ for any $0<|\varepsilon| \leq \varepsilon_{0}$.

By taking $\Omega=\mathcal{M}$, we see [7] that assumption (H3) holds if the Euler characteristic $\chi\left(\bigcup_{\tau \in \mathcal{M}} \mathcal{R}\left(I-S_{\tau}\right) \operatorname{Fix}(-R)\right)$ is nonzero. Then Theorem 4.1 holds under assumption (H1) for any $R$-reversible $C^{2}$-smooth perturbation $f(x, \varepsilon)$.

If $f(x, \varepsilon)$ is $C^{3}$-smooth, then $F$ is $C^{2}$-smooth. To solve $F(\tau, v, \varepsilon)=0$, we follow the standard way [1] by splitting it as $F(\tau, v, \varepsilon)=S_{\tau} F(\tau, v, \varepsilon)+\left(I-S_{\tau}\right) F(\tau, v, \varepsilon)$. By using the implicit function theorem, we can solve the equation $S_{\tau} F(\tau, v, \varepsilon)=0$ in $v$ for $\varepsilon$ small and $\tau \in \mathcal{M}$ to get the $C^{2}$-smooth solution $v=v(\tau, \varepsilon)=O(\varepsilon)$. Then we consider the bifurcation equation $C(\tau, \varepsilon)=\left(I-S_{\tau}\right) F(\tau, v(\tau, \varepsilon), \varepsilon)=0$. Clearly $C(\tau, \varepsilon) / \varepsilon \rightarrow B(\tau)$ in the $C^{1}$-topology on $\mathcal{M}$ as $\varepsilon \rightarrow 0$. Consequently, a simple zero $\tau_{0}$ of $B(\tau)$, i.e. $B\left(\tau_{0}\right)=0$ and $D B\left(\tau_{0}\right)$ is nonsingular, implies the solvability of $C(\tau, \varepsilon)=0$ in $\tau$ near $\tau_{0}$ for $\varepsilon \neq 0$ small. Summarizing we see that $B$ is the Melnikov function for this problem, since its simple zero $\tau_{0}$ ensures the bifurcation of an $R$-symmetric homoclinic orbit of $f(x, \varepsilon)$ to $p_{\varepsilon}$ for $\varepsilon \neq 0$ small bifurcating from the $R$-symmetric homoclinic orbit of $f(x, 0)=f(x)$ which starts from $\tau_{0} \in \mathcal{M}$.

\section{AN EXAMPLE}

In this section, we present an example, but first we simplify the formula of $B(\tau)$. If $a \in \mathcal{R}\left(I-S_{\tau}\right)$, then $a \in \operatorname{Fix}(-R)$ and $a \perp(I-R) T_{\tau} \widetilde{W}_{p}^{s}$. Hence for any $w \in T_{\tau} \widetilde{W}_{p}^{s}$ we have

$$
0=\langle a,(I-R) w\rangle=\langle a, w\rangle-\langle a, R w\rangle=\langle a-R a, w\rangle=2\langle a, w\rangle .
$$

Furthermore, since $R T_{\tau} \widetilde{W}_{p}^{s}=T_{R \tau} \widetilde{W}_{p}^{u}=T_{\tau} \widetilde{W}_{p}^{u}$ and for any $w \in T_{\tau} \widetilde{W}_{p}^{s}$, we have

$$
\langle a, R w\rangle=-\langle R a, R w\rangle=-\langle a, w\rangle=0,
$$

we see that $a \in \mathcal{R}\left(I-S_{\tau}\right)$ if and only if $a \perp\left(T_{\tau} \widetilde{W}_{p}^{s}+T_{\tau} \widetilde{W}_{p}^{u}+\right.$ Fix $\left.R\right)$. We note that $\operatorname{Fix}(-R)=(\operatorname{Fix} R)^{\perp}$, and $\frac{1}{2}(I-R): \mathbb{R}^{2 N} \rightarrow \operatorname{Fix}(-R)$ and $\frac{1}{2}(I+R): \mathbb{R}^{2 N} \rightarrow$ Fix $R$ are the orthogonal projections. Consequently, if $a_{i}(\tau), i=1,2, \cdots, \operatorname{dim} \mathcal{M}$, is a continuous orthonormal vector field such that $a_{i}(\tau) \perp\left(T_{\tau} \widetilde{W}_{p}^{s}+T_{\tau} \widetilde{W}_{p}^{u}+\mathrm{Fix} R\right)$, then the components of $B(\tau)$ are given by

$$
B_{i}(\tau)=\left\langle a_{i}(\tau),(I-R) D_{\epsilon} x_{0}^{+}(\infty, \tau, 0)\right\rangle=2\left\langle a_{i}(\tau), D_{\epsilon} x_{0}^{+}(\infty, \tau, 0)\right\rangle .
$$

Now we consider a perturbed second order differential equation

$$
\ddot{z}=g(z)+\varepsilon h(z), \quad z \in \mathbb{R}^{N},
$$

where $g, h \in C^{3}\left(\mathbb{R}^{N}, \mathbb{R}^{N}\right), g(0)=h(0)=0$. (8) has the form

$$
\dot{z}_{1}=z_{2}, \quad \dot{z}_{2}=g\left(z_{1}\right)+\varepsilon h\left(z_{1}\right) .
$$

Let $\phi\left(t, z_{1}, z_{2}, \varepsilon\right)$ be the flow of (9), then $f(x, \varepsilon)=\phi(T, x, \varepsilon), x=\left(z_{1}, z_{2}\right)$ for a $T>0$. Here $R\left(z_{1}, z_{2}\right)=\left(z_{1},-z_{2}\right)$ and Fix $R=\left\{\left(z_{1}, 0\right) \mid z_{1} \in \mathbb{R}^{N}\right\}$, Fix $(-R)=\left\{\left(0, z_{2}\right) \mid\right.$ $\left.z_{2} \in \mathbb{R}^{N}\right\}$. The inner product $\langle\cdot, \cdot\rangle$ is given by $\left\langle\left(z_{1}^{1}, z_{2}^{1}\right),\left(z_{1}^{2}, z_{2}^{2}\right)\right\rangle=\left(z_{1}^{1}, z_{1}^{2}\right)+\left(z_{2}^{1}, z_{2}^{2}\right)$, where $(\cdot, \cdot)$ is the usual inner product on $\mathbb{R}^{N}$. We assume that $p_{\varepsilon}=(0,0)$ is a hyperbolic equilibrium of (9). Let $\tau \in \operatorname{Fix} R \cap \widetilde{W}_{p}^{s}$. Then $\phi(t, \tau, 0)=\left(z_{1}^{\tau}(t), z_{2}^{\tau}(t)\right)$ with $z_{1}^{\tau}(t)$ even and $z_{2}^{\tau}(t)$ odd. $\phi(t, \tau, 0)$ is a homoclinic solution of (9) with $\varepsilon=0$. The linearization of (9) for $\varepsilon=0$ along $\phi(t, \tau, 0)$ has the form

$$
\dot{v}=w, \quad \dot{w}=D g\left(z_{1}^{\tau}(t)\right) v .
$$


It is clear that $T_{\tau} \widetilde{W}_{p}^{s(u)}=\{(v(0), w(0)) \mid v(t), w(t)$ are bounded solutions of (10) on $\left.\mathbb{R}_{+(-)}\right\}$, respectively. According to [2], the condition $a \perp\left(T_{\tau} \widetilde{W}_{p}^{s}+T_{\tau} \widetilde{W}_{p}^{u}+\right.$ Fix $\left.R\right)$ is now equivalent to $a=\left(0, a_{2}\right)$ and $v_{1}(0)=0, w_{1}(0)=a_{2}$, where $v_{1}(t)$ and $w_{1}(t)$ are the bounded solutions on $\mathbb{R}$ of the adjoint system of (10), i.e. $w_{1}$ is the even bounded solution on $\mathbb{R}$ of $\ddot{w}_{1}=D g\left(z_{1}^{\tau}(t)\right)^{*} w_{1}, w_{1}(0)=a_{2}$. Furthermore, $D_{\varepsilon} x_{0}^{+}(\infty, \tau, 0)=\left(v_{\tau}(0), w_{\tau}(0)\right) \perp T_{\tau} \widetilde{W}_{p}^{s}$, where $v_{\tau}(t)$ and $w_{\tau}(t)$ are the bounded solutions on $\mathbb{R}_{+}$of the equations

$$
\dot{v}_{\tau}=w_{\tau}, \quad \dot{w}_{\tau}=D g\left(z_{1}^{\tau}(t)\right) v_{\tau}+h\left(z_{1}^{\tau}(t)\right)
$$

Consequently, the corresponding component of $B(\tau)$ to $a$ derived above is given by $2\left\langle a, D_{\varepsilon} x_{0}^{+}(\infty, \tau, 0)\right\rangle=2\left\langle\left(0, a_{2}\right),\left(v_{\tau}(0), w_{\tau}(0)\right)\right\rangle=2\left(a_{2}, w_{\tau}(0)\right)=2\left(w_{1}(0), w_{\tau}(0)\right)$.

On the other hand, since (11) holds along with $\lim _{t \rightarrow+\infty} w_{1}(t)=0$, we have

$$
\int_{0}^{\infty}\left(h\left(z_{1}^{\tau}(t), w_{1}(t)\right) d t=\left(w_{1}(0), w_{\tau}(0)\right) .\right.
$$

To be more concrete, we consider the system

$$
\ddot{x}=x-2 x\left(x^{2}+y^{2}\right), \ddot{y}=y-2 y\left(x^{2}+y^{2}\right)+\varepsilon x^{4}, \quad x, y \in \mathbb{R} .
$$

(13) has for $\varepsilon=0$ a homoclinic manifold $x_{\tau}(t)=\sin \tau r(t), y_{\tau}(t)=\cos \tau r(t)$, $r(t)=\operatorname{sech} t$, which intersects Fix $R$ in the circle $\mathcal{M}=(\sin \tau, \cos \tau, 0,0)$. It is not difficult to observe that now assumption (H1) holds and $w_{1}(t)=\left(y_{\tau}(t),-x_{\tau}(t)\right)$. By (12), the function $B(\tau)$ now has the form

$$
B(\tau)=-2 \int_{0}^{\infty} \sin ^{5} \tau r^{5}(t) d t=-\frac{3}{8} \pi \sin ^{5} \tau .
$$

We note that $x_{0}(t), y_{0}(t)$ are the even solutions of (13). The bifurcation equation $C(\tau, \varepsilon)=0$ from Section 4 is now analytical. Hence $\tau=0$ is its isolated solution for $\varepsilon \neq 0$ small and fixed. The Brouwer degree of $B(\tau)$ at $\tau=0$ is -1 , so Theorem 4.1 implies the following result.

Theorem 5.1. The point $(0,1,0,0)$ is an isolated topologically transversal intersection of $W_{p_{\varepsilon}}^{s}$ and Fix $R$ for (13) with $\varepsilon \neq 0$ small. But this point is not a $C^{1}$-transversal intersection.

Proof. To prove the non- $C^{1}$-transversal intersection, we consider a $C^{3}$-perturbation of (13) given by

$$
\ddot{x}=x-2 x\left(x^{2}+y^{2}\right), \ddot{y}=y-2 y\left(x^{2}+y^{2}\right)+\varepsilon \phi_{\delta}(x), \quad x, y \in \mathbb{R},
$$

where $\delta>0$ and $\phi_{\delta}(x)=0$ if $|x| \leq \delta, \phi_{\delta}(x)=(x-\delta \operatorname{sgn} x)^{4}$ if $|x| \geq \delta$. We see that (14) has even homoclinics $x_{\tau}(t), y_{\tau}(t)$ for $|\sin \tau|<\delta$ and any $\varepsilon$. Hence $(0,1,0,0)$ is not an isolated reversible homoclinic point for the $C^{3}$-perturbation (14) with $\varepsilon \neq 0$, $\delta>0$ small of the system (13). The proof is finished.

\section{REFERENCES}

[1] Battelli, F., Fečkan, M., Chaos arising near a topologically transversal homoclinic set, preprint.

[2] Battelli, F., Fečkan, M., Subharmonic solutions in singular systems, J. Differential Equations, 132 (1996), pp. 21-45. MR 98k:34068

[3] Devaney, R., Blue sky catastrophes in reversible and Hamiltonian systems, Indiana Univ. Math. J., 26 (1977), pp. 247-263. MR 55:4275] 
[4] Devaney, R., Reversible diffeomorphisms and flows, Tran. Amer. Math. Soc., 218 (1976), pp. 89-113. MR 53:6629

[5] Devaney, R., Homoclinic bifurcations and the area-conserving Hénon mapping, J. Differential Equations, 51 (1984), pp. 254-266. MR 85k:58054

[6] Fonseca, I., Gangbo, W., Degree Theory in Analysis and Applications, Oxford Lec. Ser. Math. Appl. 2, Clarendon Press, Oxford (1995). MR 96k:47100

[7] Hirsch, M.W., Differential Topology, Springer-Verlag, New York (1976). MR 56:6669

[8] Palmer, K. J., Exponential dichotomies, the shadowing lemma and transversal homoclinic points, Dynamics Report Ser. Dynam. Systems Appl. 1 (1988), pp. 265-306. MR 89j:58060

[9] Vanderbauwhede, A., Heteroclinic cycles and periodic orbits in reversible systems, in J. Wiener \& J.K. Hale (Ed.) "Ordinary and Delay Differential Equations", Pitman Research Notes in Mathematics Series, No. 272 Pitman 1992, pp. 250-253. CMP 97:08

[10] Vanderbauwhede, A., Fiedler, B., Homoclinic period blow-up in reversible and conservative systems, Z. Angew. Math. Phys. (ZAMP), 43 (1992), 292-318. MR 93f:58209

Department of Mathematical Analysis, Comenius University, Mlynská dolina, 84248 Bratislava, Slovakia

E-mail address: Michal.Feckan@fmph.uniba.sk 